(c)2000 American Institute of Aeronautics \& Astronautics or published with permission of author(s) and/or author(s)' sponsoring organization.

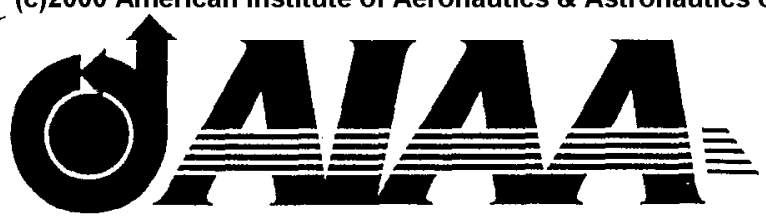

\title{
AIAA 2000-0855
}

\section{Density and Viscosity Ratio Effects in Droplet Formation}

\section{Choongil Kim \& Luis P. Bernal Department of Aerospace Engineering}

\section{University of Michigan Ann Arbor, MI 48109}

\section{8th Aerospace Sciences Meeting \& Exhibit 10-13 January 2000 / Reno, NV}


AIAA-2000-0855

\title{
DENSITY AND VISCOSITY RATIO EFFECTS IN DROPLET FORMATION
}

\author{
Choongil Kim ${ }^{\hat{s}}$, Luis P. Bernal* \\ Department of Aerospace Engineering \\ University of Michigan \\ Ann Arbor, Michigan 48109-2140
}

\begin{abstract}
New results are reported on the propagation of a vortex ring through immiscible fluid interfaces in the limit of high Weber number in microgravity and in $1-G$. The main objective of the tests is to determine the effect of density and viscosity ratios on the dynamics of the interaction. It is shown that density ratio plays a controlling role in determining the outcome of the interaction. For small density ratio, i.e. a gas/liquid interface, the vortex ring forms a long column before the interface breaks. In contrast, for a density ratio of order one, i.e. liquids of matched density, the vortex ring propagates through the interface and forms a drop. The effect of viscosity ratio for density ratio of order one is to change the size and structure of the liquid column during drop formation and the number and location of satellite droplets formed. These results suggest that density ratio effects are more important than previously recognized in computational and experimental studies of multiphase flows.
\end{abstract}

\section{INTRODUCTION}

Detailed understanding of the dynamics of liquid atomization is limited because of the scales normally associated with the atomization process. Many important atomization processes have as the ultimate goal to produce very small drops. For example in liquid fuel atomizers for internal combustion engines and in spray atomizers for drug delivery in biomedical systems, the goal is to produce very small droplets to facilitate evaporation of the liquid. Another example is droplet formation in inkjet print heads. The size of the droplet determines the resolution of the printer. Smaller droplet size gives better resolution and print quality. These systems operate in the regime of surface tension dominated interface breakup because of the small size of the droplets. These cases are also

\footnotetext{
\$ Graduate Research Assistant, Department of Aerospace

Engineering. Member AIAA.

* Assuciate Professor, Department of Aerospace Engineering, Senior Member AIAA.

Copyright $\stackrel{\oplus}{\mathbf{C}} 2000$ Luis P Bernal, Published by the American Institute of Aeronautics and Astronautics, Inc. with permission
}

characterized by a very small density ratio because the liquid drops form in air that gives a density ratio of the order of $10^{-3}$. Attempts to study these phenomena at larger scale are hindered by the fact that the relative magnitude of surface tension and the gravitational force
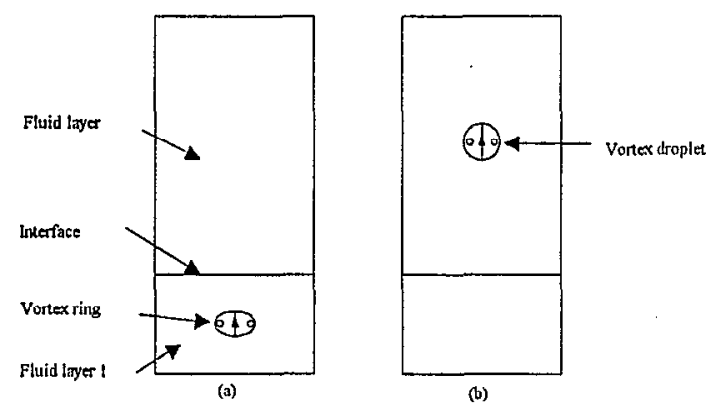

Figure 1. Schematic diagram of the interaction of a vortex ring with a fluid interface. (a) Illustration of the flow before the interaction showing the vortex ring before it reached the interface. (b) Illustration of the flow after interaction showing the formation of a vortex droplet

changes with the size of the droplet. As the size of the droplet increases the gravitational force becomes increasingly more important.

In order to recover the surface-tension-dominated interface dynamics in larger scale tests it is necessary to minimize the effect of the gravitational force. This can be accomplished by testing under actual microgravity conditions (the preferred testing environment) or by matching the density of the fluids (simulated microgravity conditions). Also computational studies of these flow processes are frequently conducted for density ratio of order one because it facilitates the numerical solution of the problem. The role of density and viscosity ratio on interface dynamics is not well understood. Density ratio effects could change in a fundamental way the liquid breakup process. This would invalidate the premise of simulated microgravity testing for liquid breakup experiments. 
In this paper we study the interaction of a vortex ring with the interface betwcen two immiscible liquids. Earlier experiments in I-G with density ratio of order one to simulate microgravity conditions, show that when the Froude and Weber numbers are high the vortex ring propagates through the interface and forms a droplet. ${ }^{2}$ Figure 1 shows a schematic diagram of the phenomenon. Here we report new microgravity tests conducted in the 2.2 second drop-tower facility at NASA Glenn under actual microgravity conditions, and additional tests in $1-G$ to clarify the role of density ratio and viscosity ratio on the evolution of the interface and the liquid breakup process.

\section{FLOW FACWITY AND EXPERIMENTAL TECHNIQUES}

The microgravity experiments were conducted in the 2.2-second drop tower at NASA Glenn. The drop tower rig is shown in Figure 2. It is equipped perform both flow visualization experiments and PIV measurements. Only flow visualization results are reported here. To visualize the flow, a new sheet of panel light was used to illuminate the fluid test cell. The measurements of the position of the vortex ring and of the deformation of interface were recorded in standard video format. The video recordings were digitized and analyzed. The fluid test cell is $15 \mathrm{~cm} \times 15$ $\mathrm{cm} \times 300 \mathrm{~cm}$. A piston-type vortex ring generator is attached to the bottom of the test cell. The motion of a piston inside a cylinder produces the vortex ring. At the end of the piston stroke the piston surface is flush with the bottom of the container resulting in a smooth flat surface during the test. A pneumatic actuator drives the piston. The input air pressure to the pneumatic actuator determines the speed of the piston and consequently the initial speed, $U$, of the vortex ring. A pressure regulator and a solenoid valve were used to control the input air pressure and the start of the fluid motion. The drop tower ring is self-contained. An air bottle carries pressurized air to drive the pneumatic actuator. Also shown in figure 2 are the two battery packs used to power the experiment during the drop. A control box detects the release of the drop tower rig and initiates the vortex ring motion and starts a timing light shown in all the video recordings.

The same apparatus was used in the 1-G tests reported here. In the 1-G tests the flow visualization images were recorded using a high-speed motion analyzer.

\section{RESULTS AND DISCUSSION}

For the microgravity tests, the flow evolution depends on fluid inertia, viscosity and surface tension. Therefore, the non-dimensional numbers defining the problem are the Weber number, and the Ohnesorge number, as well as the density and viscosity ratios.

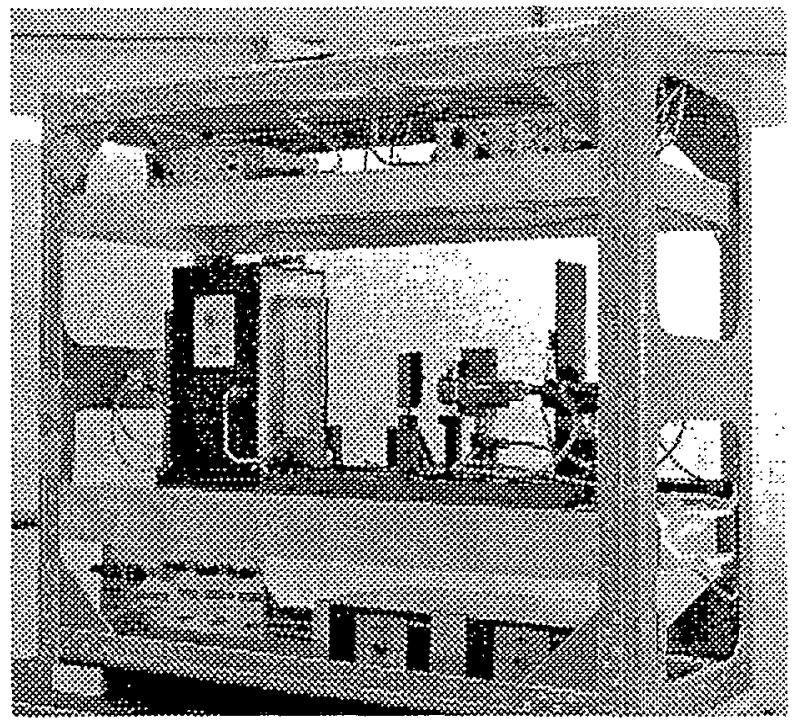

Figure 2. Drop Tower rig

The Weber, We, is defined based on the vortex ring circulation and is given by

$$
W e=\frac{\rho_{1} \Gamma^{2}}{\sigma a}
$$

It gives the ratio of inertia to surface tension forces. Here $\rho_{1}$ is the density of the fluid in the bottom layer, $\Gamma$ is the circulation of the vortex ring, $\sigma$ is the interfacial tension and $a$ is the vortex ring diameter.

The Ohnesoge number characterizes the relative magnitude of viscous forces compared to surface tension force. It is defined as,

$$
O h=\frac{\mu_{1}}{\sqrt{\rho_{1} \sigma a}},
$$

with $\mu_{1}$ the viscosity of the fluid in the bottom layer. The Reynolds number is defined as

$$
\operatorname{Re}=\frac{\rho_{1} \Gamma}{\mu_{1}}=\frac{\sqrt{\mathrm{We}}}{\text { Oll }}
$$

Finally, the density ratio, $r$, and the viscosity ratio, $v$. are given by

$$
\mathrm{r}=\frac{\rho_{2}}{\rho_{1}}, \quad \mathrm{v}=\frac{\mu_{2}}{\mu_{1}},
$$

where subscript 1 refers to the fluid in the bottom layer and subscript 2 refers to the top fluid layer.

In 1-G experiments the Bond number characterizes the relative magnitude of gravitational force. The Bond number gives the ratio of gravitational force to surface tension force and is given by,

$$
\mathrm{Bo}=\frac{\left(\rho_{1}-\rho_{2}\right) \mathrm{ga}^{2}}{\sigma},
$$

where $\mathrm{g}$ is the gravitational acceleration. 
Figure 3 shows the interaction of a vortex ring with an interface in microgravity. The fluid in the bottom layer is 2 centistokes silicon oil and the fluid in the top layer is air. The Weber number 220 , and Ohnesorge number is 0.003 . In this case the geometrical features of the vortex ring disappear quickly and the interaction results in a long liquid column. The thickness of the column is significantly less than the diameter of the vortex ring. The liquid surface breaks much later in time at the bottom of the column. A small satellite droplet forms at the bottom of the column after interface breakup and there are indications that additional droplets might form. Figure 4. Shows the interaction at the same flow conditions except for the fluid in the bottom layer that is now $10 \mathrm{cs}$ silicon oil. In this case the Weber number is 94 and the Ohnesorge number is 0.016 . The interaction has essentially the same features as in the previous case at the higher Weber number. However, interface breakup occurs at a later compared to the $2 \mathrm{cs}$ case. This is probably due to the higher viscosity. In these cases the Reynolds number are 4720 for the $2 \mathrm{cs}$ case and 617 for the $10 \mathrm{cs}$ case.

Figure 5 and Figure 6 show the interaction at higher piston velocity with the same liquids as in the cases shown in figure 3 and 4 . The Weber number in these cases are 447 for the $2 \mathrm{cs}$ case (Figure 5) and 500 for the $10 \mathrm{cs}$ case. The main features of the interaction are essentially the same as in the lower velocity case. The only difference is the column length before interface breakup is longer in this case. Another clifference is that larger satellite droplets from at the bottom of the column. These results show that above a critical Weber number the main interaction features are not strongly dependent on the Weber number. The critical Weber number is the value associated with interface breakup.

The effect of density ratio on the interaction is shown in Figures 7 and 8 . Figure 7 shows the interaction at low density ratio $r=0.00143$ and a Reynolds number of approximately 4720 . Figure 8 shows the interaction at a density ratio $r=0.785$ and Reynolds number of approximately 4800 . The interaction shown in Figure 8 is for the interface between air and 2 cs silicon oil, the same as in Figure 3. In contrast. Figure 8 shows the interaction for the interface between a mixture of glycerol and water on top and $2 \mathrm{cs}$ silicon oil at the bottom. For the case of density ratio of order one (Figure 8) the vortex ring propagates through the interface and retains the main features. A liquid column of fluid from the bottom layer trails the vortex ring. The liquid column breaks at two locations and forms a main droplet on top that contains the fluid (and vorticity) of the initial vortex ring, and a satellite drop. In comparing the cases in
Figures 7 and 8 it should be noted that the viscosity ratio is also different. For the interaction shown in Figure 7 the viscosity ratio is $v=0.0$ land for the case shown in Figure 8 the viscosity ratio is 0.39 . It is not clear therefore whether the differences in interaction dynamics are due to viscosity ratio or, more likely. density ratio. The evolution shown in Figure 8 is very similar to the interaction observed in 1-G experiments with liquid layers of almost-matched densities." ? Clearly those 1-G experiments do not capture the interface evolution found at very low density ratio.

To further examine this issue, Figures 9 and 10 show a comparison between cases the same fluid interface and vortex ring circulation at microgravity (figure 10) and 1-G (figure 9). The Weber number in these cases is 2070 , larger that the critical value, the Reynolds number is 9500 , the density ratio is $r=0.873$, and the viscosity ratio is $v=1.34$. The interactions in these cases are very similar. The only apparent difference is the thickness of the liquid column below the main vortex, the $1-G$ condition giving the thicker column. Also the details of interface breakup and formation of satellite droplets are different in the two cases. We conclude than that the $1-G$ experiments accurately capture the interface evolution at microgravity conditions.

To clarify the role of density ratio and viscosity ratio on interface dynamics experiments were conducted with liquid layers having similar density ratio (of order one) and varying viscosity ratio. These results are shown in Figures 11 and 12. Figure: 11 shows the interaction with a methanol-water mixture in the bottom layer and $10 \mathrm{cs}$ silicon oil on the top layer. The parameters of the interaction are: $r=0.97$, $v=0.187, R e=616$ and $W e=100$. Figure 13 shows the interaction with $10 \mathrm{cs}$ silicon oil on the bottom layer and water on the top layer. The parameters of the interaction are: $r=0.94, v=7.231, R e=4800$ and $\mathrm{We}=222$. These experiments were conducted at $1-\mathrm{G}$. The interaction for small viscosity ratio shows formation of a large droplet. The column below the drop persists a short time. For the case of large viscosity ratio the vortex ring propagates through the interface into the top fluid layer. A more persistent column forms with interface breakup at the bottom of the column.

Figures 13 and 14 shown the same liquid pair systems as in Figures 11 and 12, respectively. but the experiments are performed at a higher Reynolds number also in 1-G conditions. For the interaction shown in Figure 13 the flow parameters are: $r=0.97$. $v=0.187, \operatorname{Re}=1722$ and $W e=780$. For the interaction shown in Figure 14 the flow parameters are: $r=0.94$. $v=7.231, \operatorname{Re}=8700$ and $\mathrm{We}=721$. In both of these cases the interaction results in the formation of a 


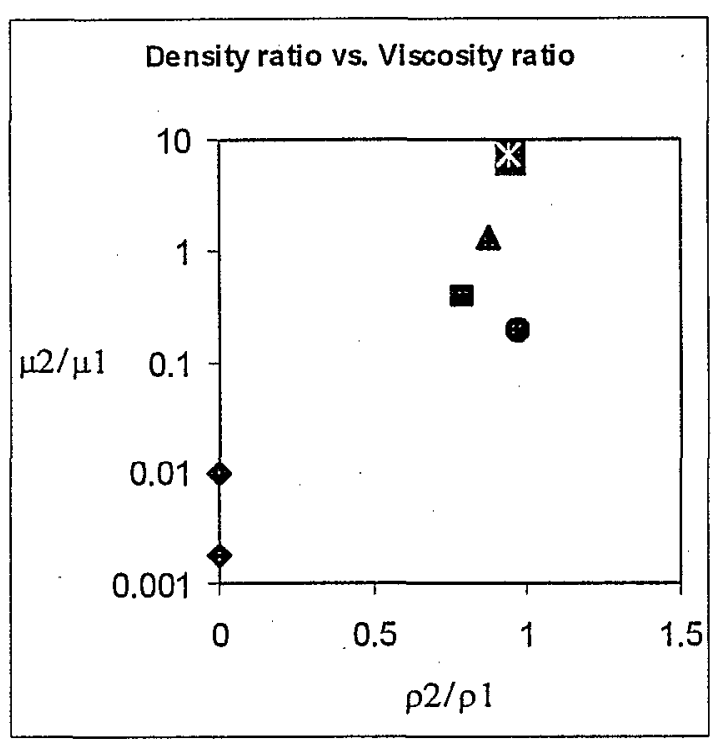

Figure 15. Viscosity ratio - density ratio map showing the flow conditions studied in this investigation.

droplet that contains the fluid and vorticity in the initial vortex ring. The evolution of the fluid column below the main droplet differs in the two cases. For viscosity ratio less than one the liquid column breaks from the main drop very early in the process and collapses to the lower liquid layer without forming satellite droplets. For viscosity ratio greater than one the liquid column persists for a longer time and breaks at several locations to form several satellite drops. These results show that a change in viscosity ratio by a factor of 50 does not result in the elongated liquid column found at lowdensity ratio in the microgravity tests.

The flow conditions studied in this investigation are summarized in the v-r map shown in Figure 15. Each point in this plot corresponds to a different fluid pair. The diamond symbols on the left of the plot correspond to the air-silicon oil interface. The interaction in these two cases results in the formation of a long column. Droplets form at a later time. Column instabilities are observed that result in interface breakup and the formation of multiple satellites drops. In these cases the droplets form with a diameter significantly smaller than the initial vortex rig diameter. The effect of viscosity ratio is small and the small changes observed can be attributed to the change in Reynolds number.

The points in Figure 15 for density ratio of order one include fluid pairs that were tested at $1-G$ and in microgravity. In these cases the propagation of the vortex ring through the interface results in the formation of a main droplet that retains the approximate shape of the vortex ring. A liquid column forms below the main droplet. Also in these cases column instabilities result in the formation of satellite droplets. In these cases the main droplet diameter is of the order of the initial vortex ring diameter and the satellite droplets form with a smaller diameter. The effect of viscosity ratio is to alter the details of the liquid columm formation and its instability.

\section{CONCLUSION}

The interaction of a vortcx ring with the interfacc between two immiscible liquids in microgravity was investigated experimentally in drop tower test at NASA Glenn Research Center and in I-G experiments. The results show that density ratio strongly influence the dynamics of the interaction. For gas liquid interface with low density ratio the interaction results in a long liquid column that break into one or more small droplets. The diameter of the droplets is significantly less than the initial vortex ring diameter. In contrast, for a density ratio of order one, i.e. liquids of matched density, the vortex ring propagates through the interface and forms a drop of substantially the same diameter. The effect of viscosity ratio for density ratio of order one is to change the size and structure of the liquid column during drop formation and the number and size of satellite droplets formed. These results suggest that density ratio effects are more important than previously recognized in computational and experimental studies of inultiphase flows.

\section{ACKNOWLEDGEMENTS}

This research was sponsored by NASA Microgravity Fluid Physics program. Grant no. NCC3474. The support provided by Dr. John McQuillen for the drop tower tests is greatly appreciated.

\section{REFERENCES}

1 L.P. Bernal, P. Maksimovic, F. Tounsi \& G. Tryggvason. "An experimental and numerical investigation of drop formation by vortical flows in microgravity." AIAA paper 94-0244, AIAA $32^{\text {nd }}$ Aerospace Sciences Meeting \& Exhibit. Reno, NV. January 10-13, 1994.

${ }^{2}$ L.P. Bernal, P. Maksimovic. "The propagation of a vortex ring through multiple liquid interfaces in microgravity." AIAA paper 96-0593, AIAA $34^{\text {th }}$ Aerospace Sciences Meeting \& Exhibit. Reno. NV. January 15-18, 1996 


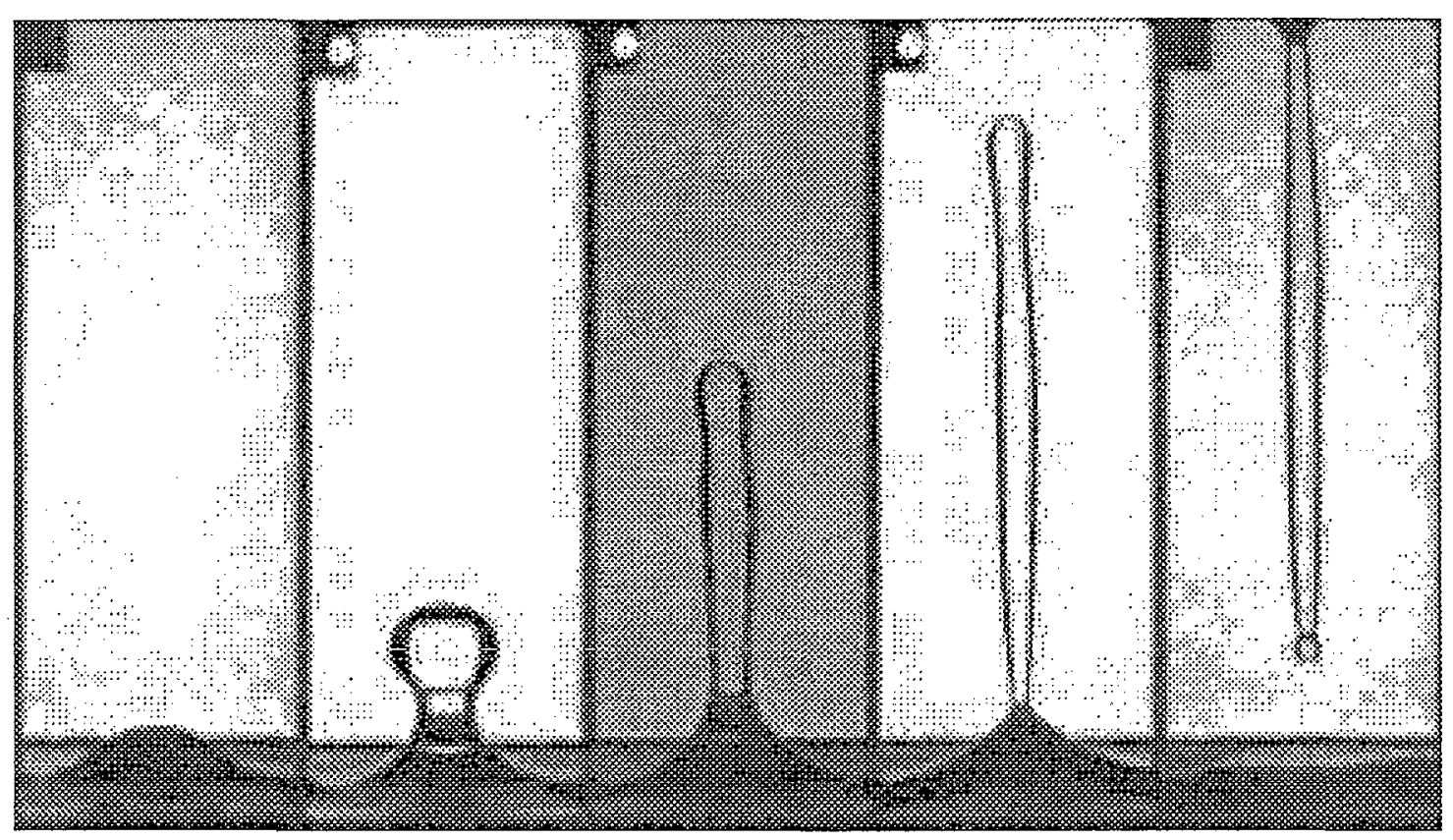

Figure 3. Top layer: Air - Bottom layer: Silicon Oil $2 \mathrm{cs}$ at $\mu-\mathrm{G}$ $\rho 2 / \rho \mathrm{l}=0.00143, \mu 2 / \mu \mathrm{l}=0.01, \operatorname{Re} \approx 4720, \mathrm{We} \approx 220, \mathrm{Oh} \approx 0.003, \Delta \mathrm{t} \approx 0.17 \mathrm{sec}$

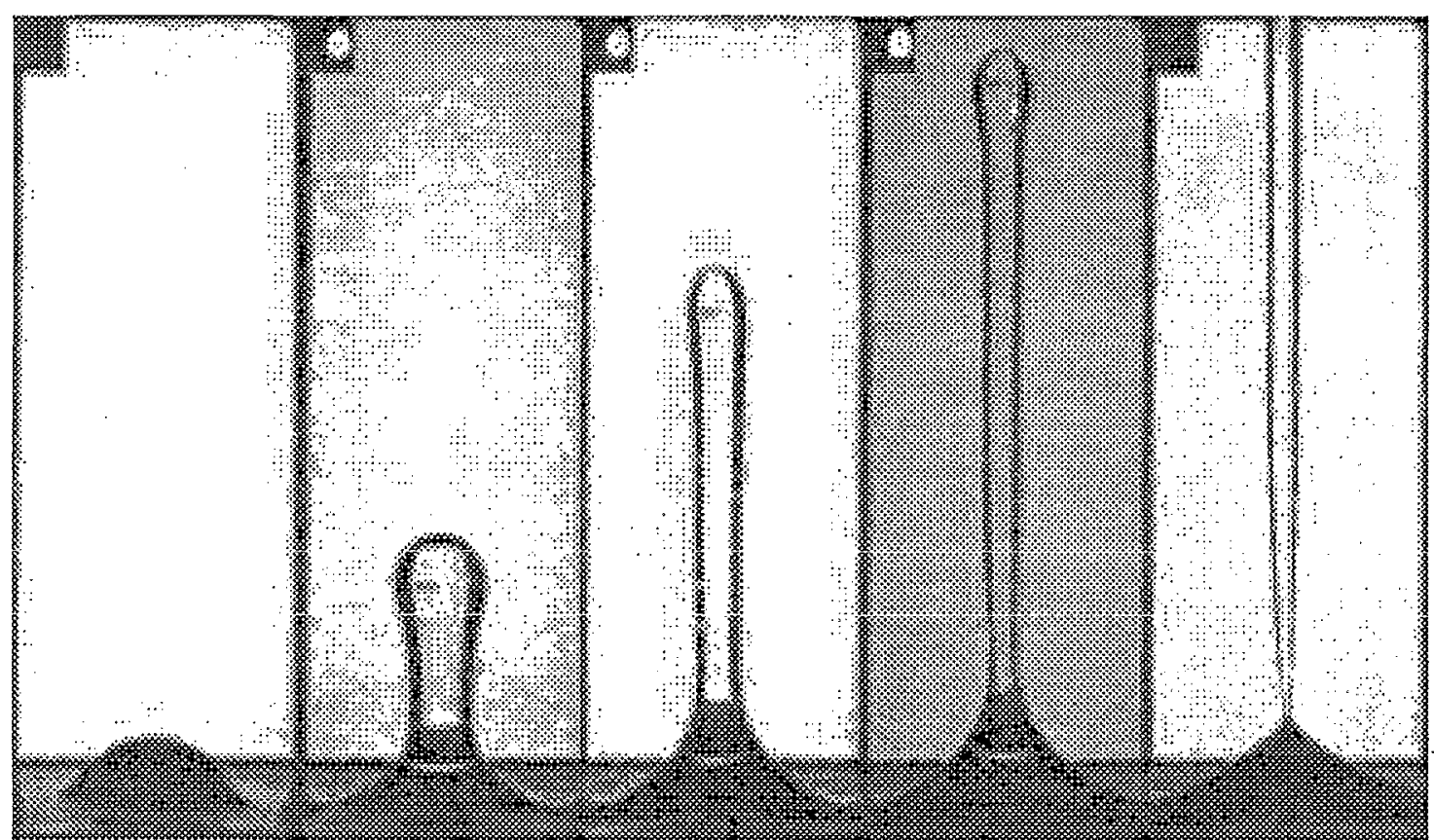

Figure 4. Top layer: Air - Bottom layer: Silicon Oil $10 \mathrm{cs}$ at $\mu-\mathrm{G}$ $\rho 2 / \rho 1=0.0013, \mu 2 / \mu 1=0.0018, \operatorname{Re} \approx 617, \mathrm{We} \approx 94, \mathrm{Oh} \approx 0.016, \Delta \mathrm{t} \approx 0.17 \mathrm{sec}$ 


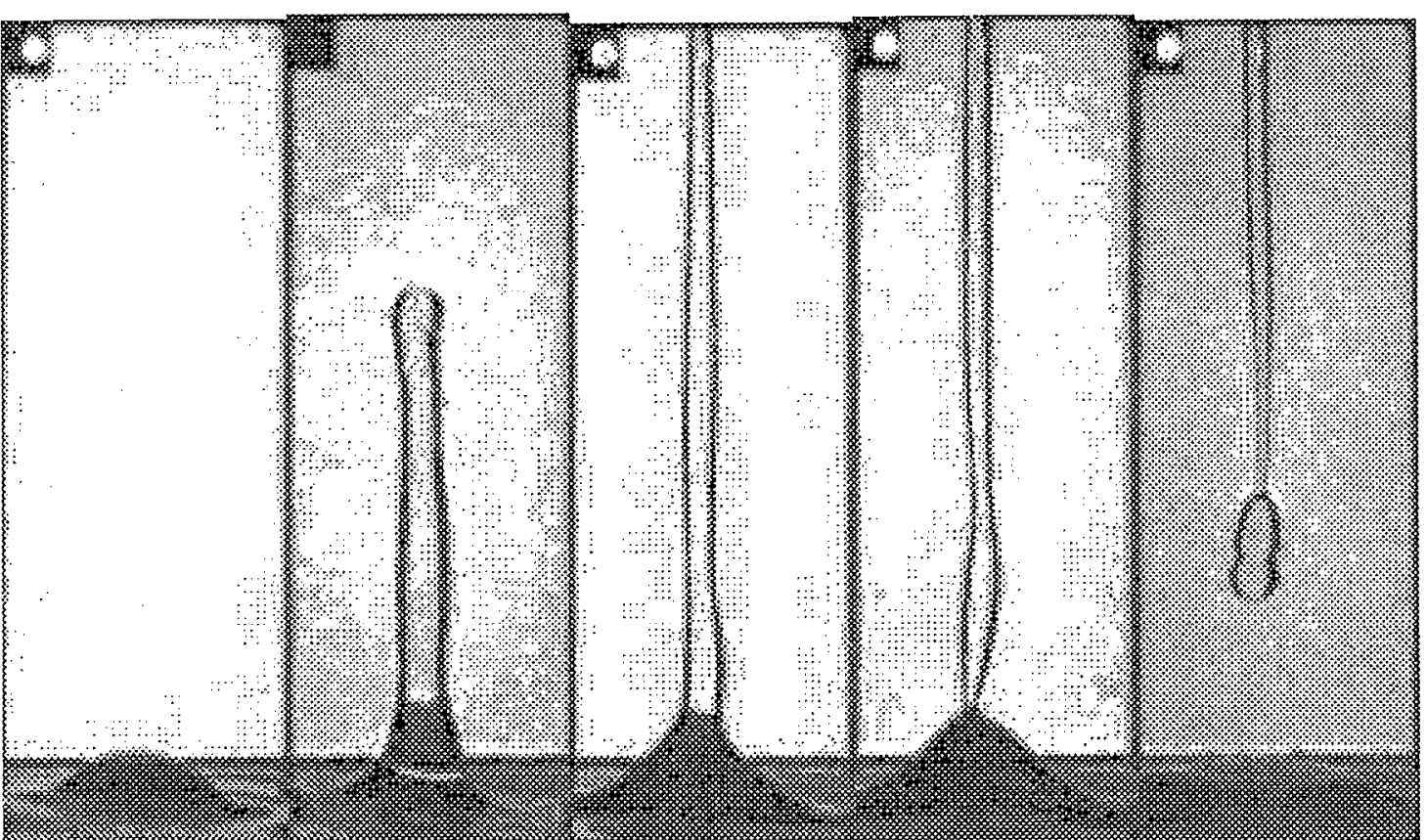

Figure 5. Top layer: Air - Bottom layer: Silicon Oil $2 \mathrm{cs}$ at $\mu-\mathrm{G}$ $\ldots \rho 2 / \rho 1=0.00143, \mu 2 / \mu 1=0.01, \operatorname{Re} \approx 6740, \mathrm{We} \approx 447, \mathrm{Oh} \approx 0.003, \Delta \mathrm{t} \approx 0.17 \mathrm{sec}$

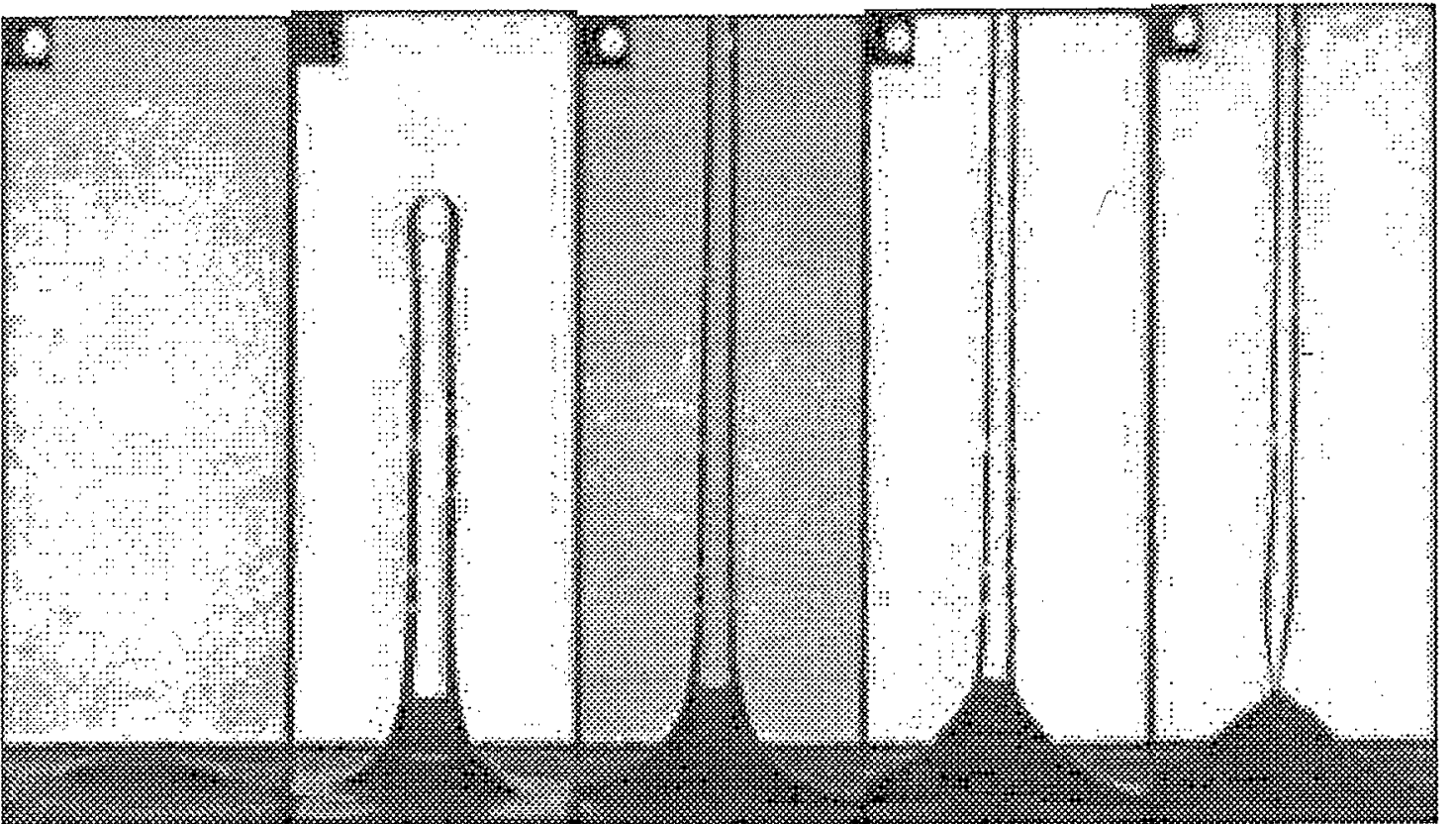

Figure 6. Top layer: Air - Bottom layer: Silicon Oil $10 \mathrm{cs} \mu-\mathrm{G}$ $\rho 2 / \rho 1=0.0013, \mu 2 / \mu 1=0.0018, \mathrm{Re} \approx 1440$, We $\approx 500, \mathrm{Oh} \approx 0.016, \Delta \mathrm{t} \approx 0.17 \mathrm{sec}$ 


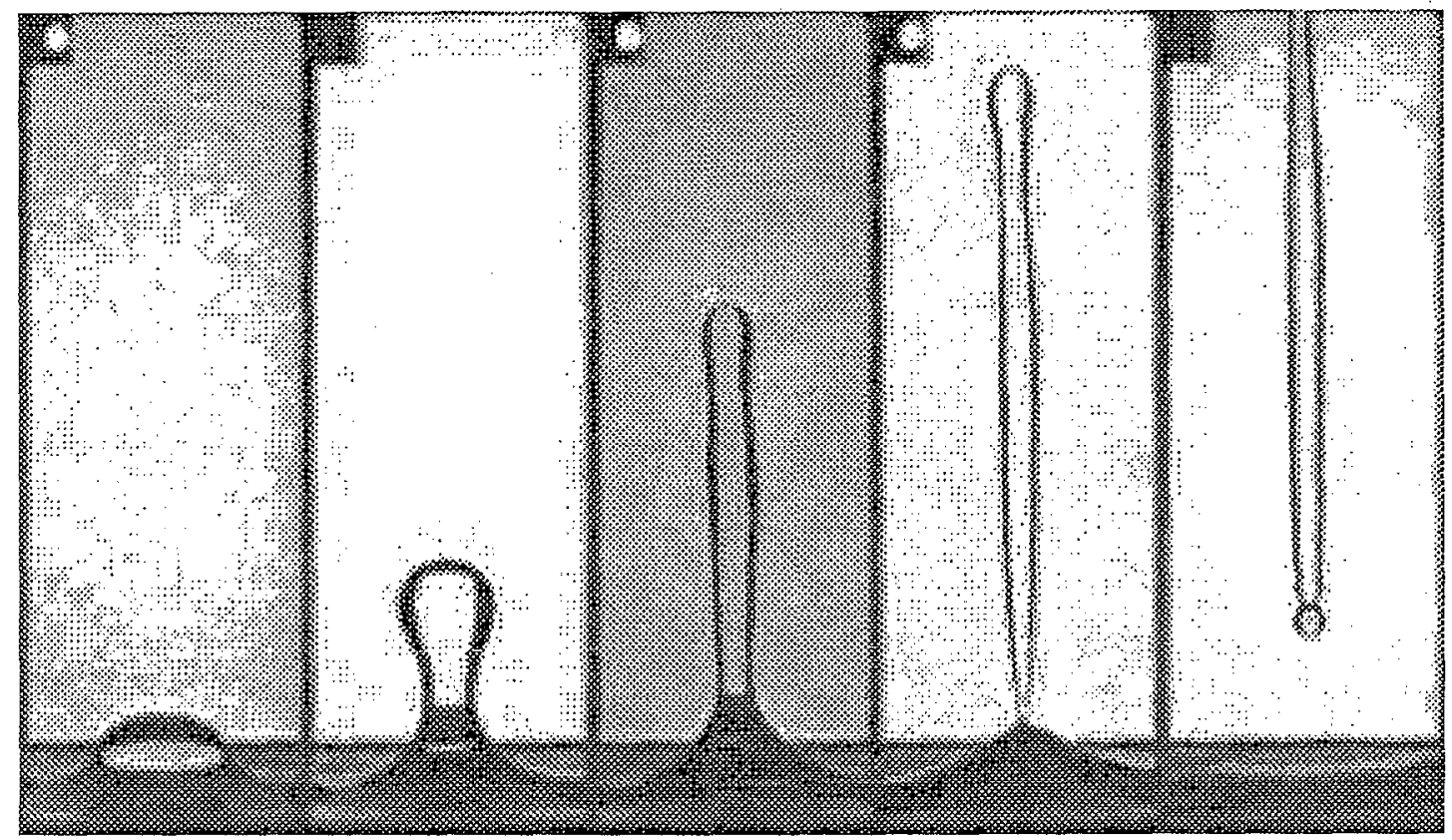

Figure 7. Top layer: Air - Bottom layer: Silicon Oil 2 cs at $\mu-G$

$\rho 2 / \rho 1=0.00143, \mu 2 / \mu 1=0.01, \operatorname{Re} \approx 4720, \mathrm{We} \approx 220, \mathrm{Ol} \approx 0.003, \Delta t \approx 0.17 \mathrm{sec}$

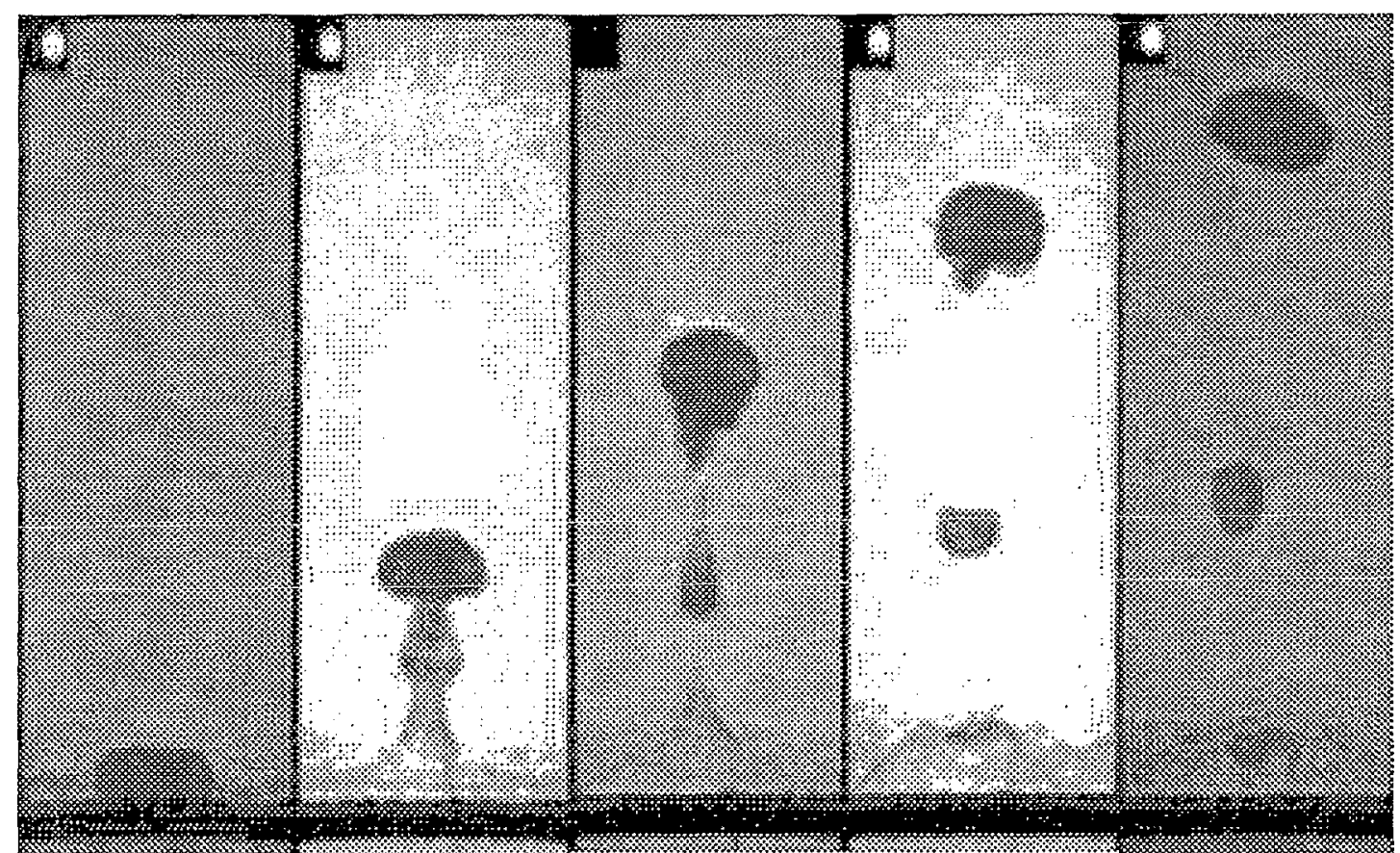

Figure 8. Top layer: Silicon Oil $2 \mathrm{cs}-$ Bottom layer: Glycerol,H2O Solution at $\mu-\mathrm{G}$ $\rho 2 / \rho 1=0.785, \mu 2 / \mu 1=0.39, \operatorname{Re} \approx 4800, \Delta t \approx 0.17 \mathrm{sec}$ 


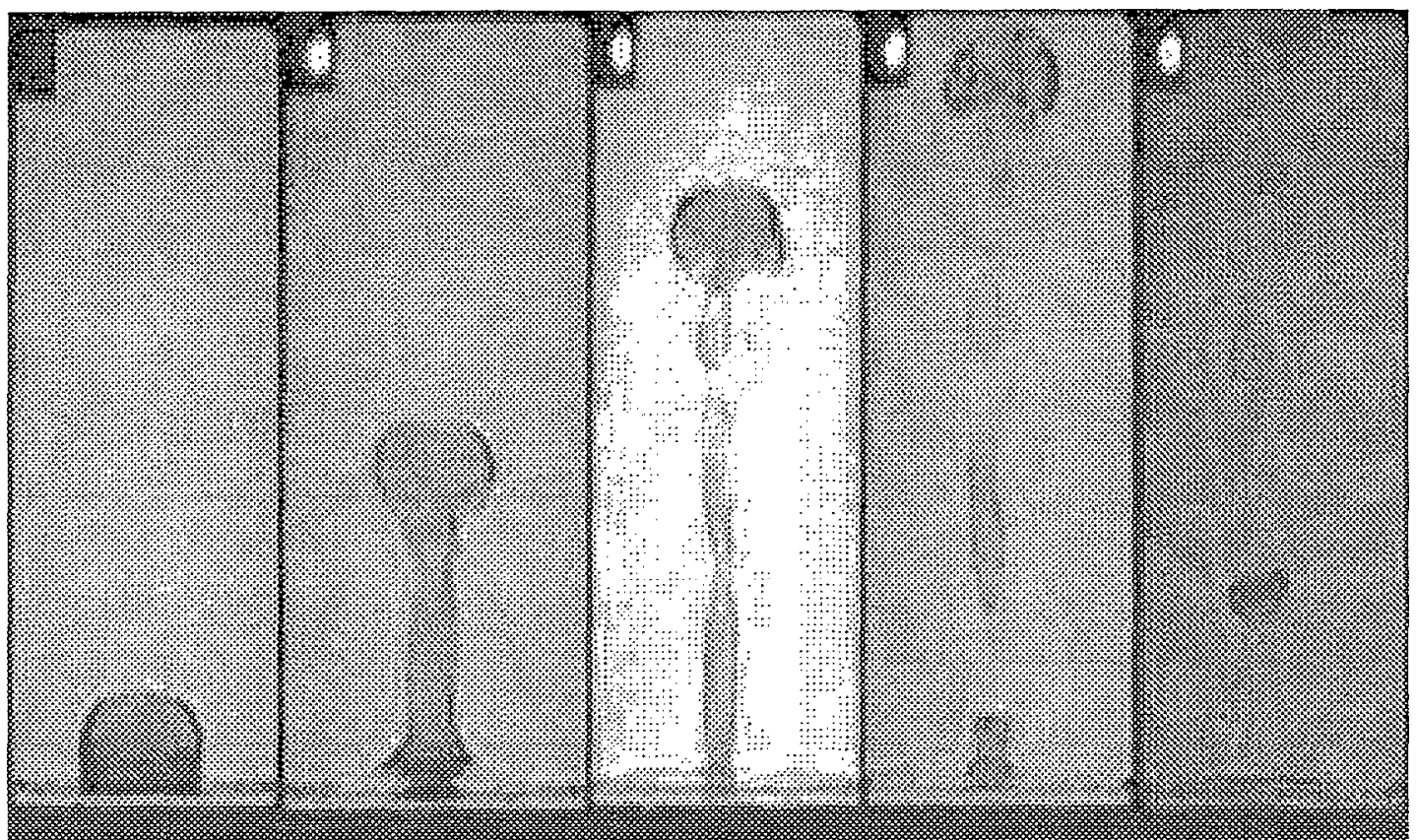

Figure 9. Top layer: Silicon Oil $2 \mathrm{cs}-$ Bottom layer: $\mathrm{H} 2 \mathrm{O}$ at $1-\mathrm{G}$ $\rho 2 / \rho 1=0.873, \mu 2 / \mu 1=1.34, \operatorname{Re} \approx 9500, \mathrm{We} \approx 2070, \mathrm{Oh} \approx 0.003, \Delta \mathrm{t} \approx 0.1 \mathrm{sec}$

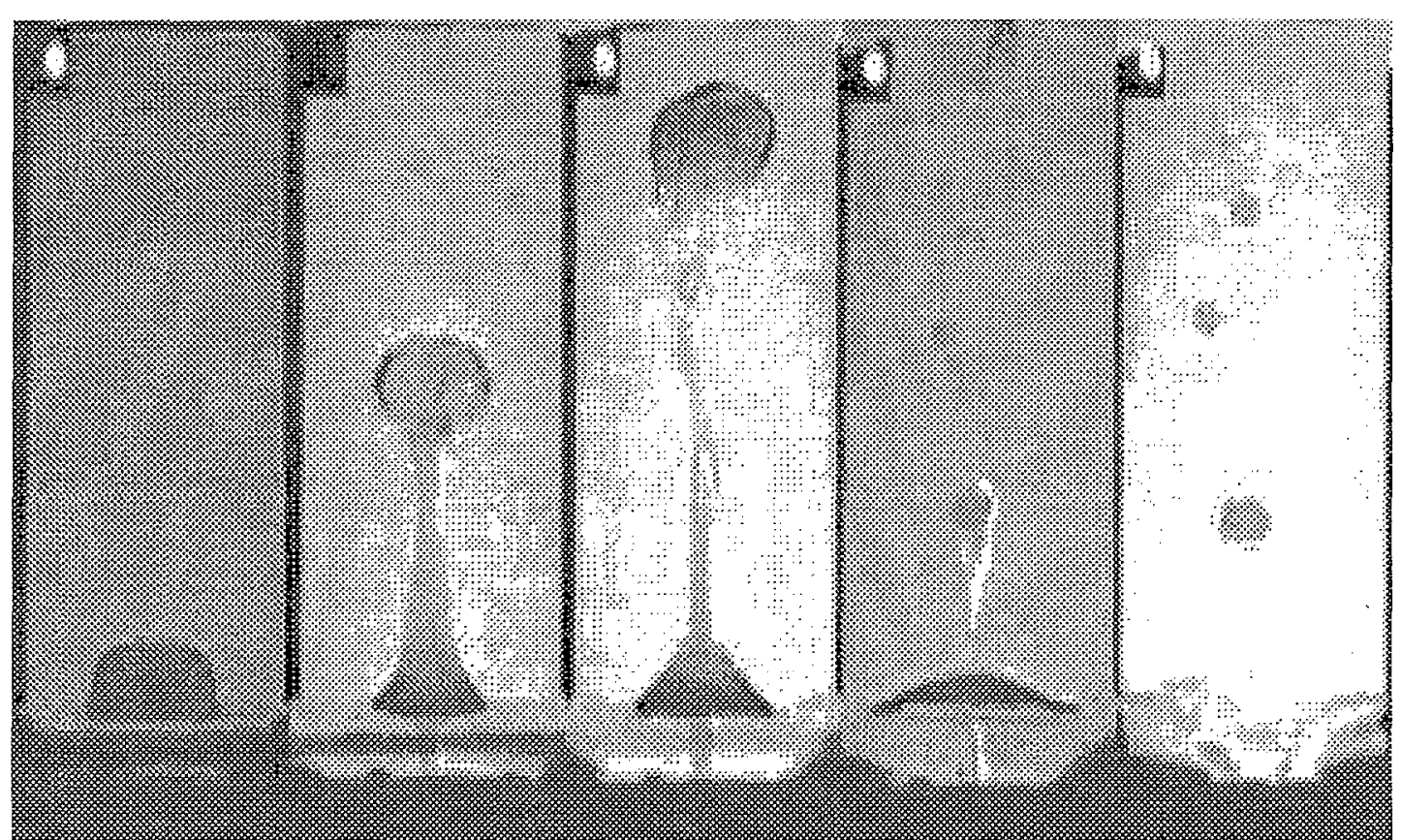

Figure 10. Top layer: Silicon Oil $2 \mathrm{cs}-$ Bottom layer: $\mathrm{H} 2 \mathrm{O}$ at $\mu-\mathrm{G}$ $\rho 2 / \rho \mathrm{I}=0.873, \mu 2 / \mu \mathrm{l}=1.34, \mathrm{Re} \approx 9500, \mathrm{We} \approx 2070, \mathrm{Oh} \approx 0.003, \Delta \mathrm{t} \approx 0.1 \mathrm{sec}$ 


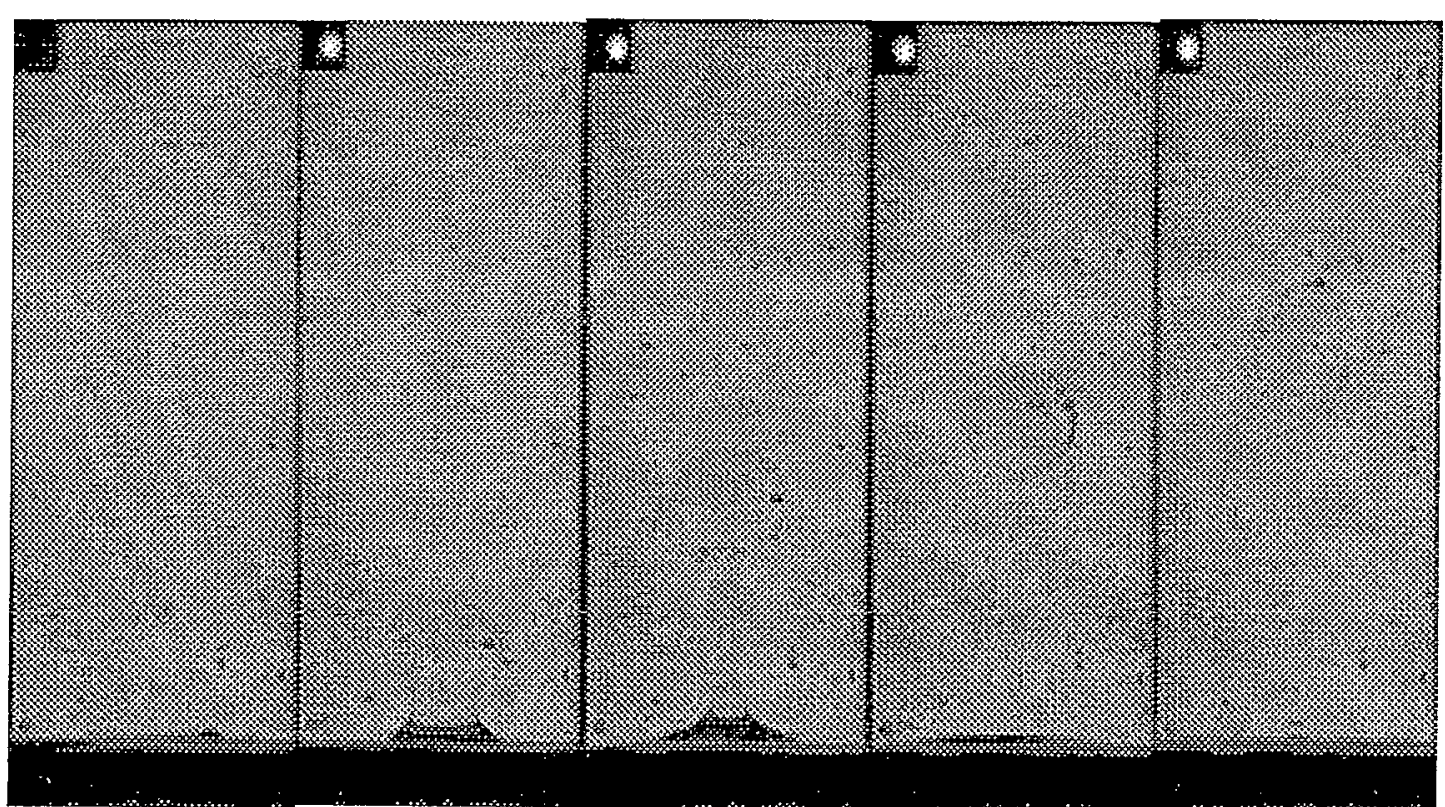

Figure 11. Top layer: Methanol, $\mathrm{H}_{2} \mathrm{O}$ solution - Bottom layer: Silicon Oil $10 \mathrm{cs}$, at $1-\mathrm{G}$ $\rho 2 / \rho \mathrm{I}=0.97, \mu 2 / \mu \mathrm{l}=0.187, \operatorname{Re} \approx 616, \mathrm{We} \approx 100, \mathrm{Oh} \approx 0.016, \Delta \mathrm{t} \approx 0.22 \mathrm{sec}$

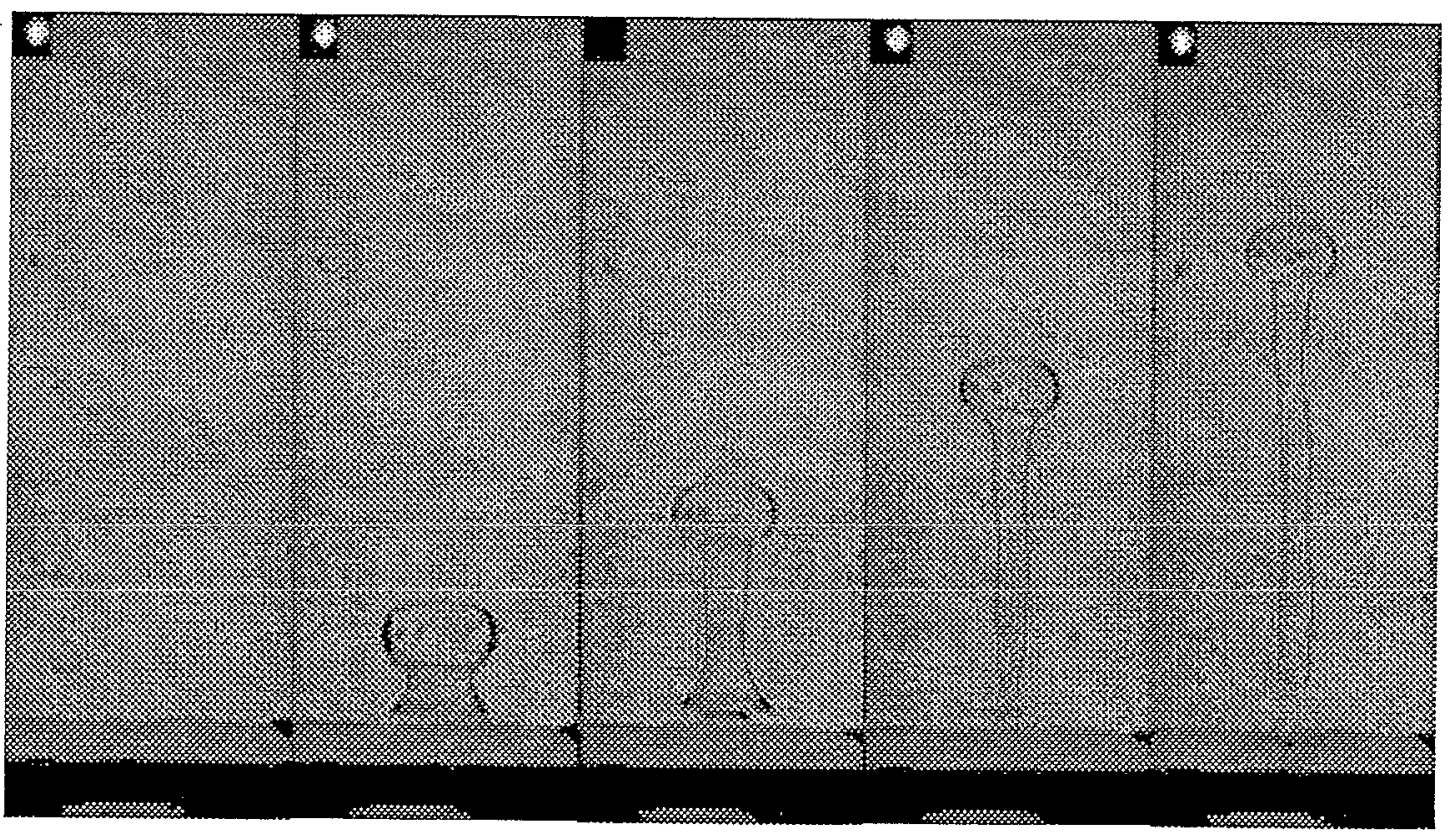

Figure 12. Top layer: Silicon Oil $10 \mathrm{cs}-$ Bottom layer: $\mathrm{H}_{2} \mathrm{O}$, at $1-\mathrm{G}$ $\rho 2 / \rho \mathrm{l}=0.94, \mu 2 / \mu \mathrm{l}=7.231, \mathrm{Re} \approx 4800, \mathrm{We} \approx 222, \mathrm{Oh} \approx 0.0019, \Delta \mathrm{t} \approx 0.22 \mathrm{sec}$ 


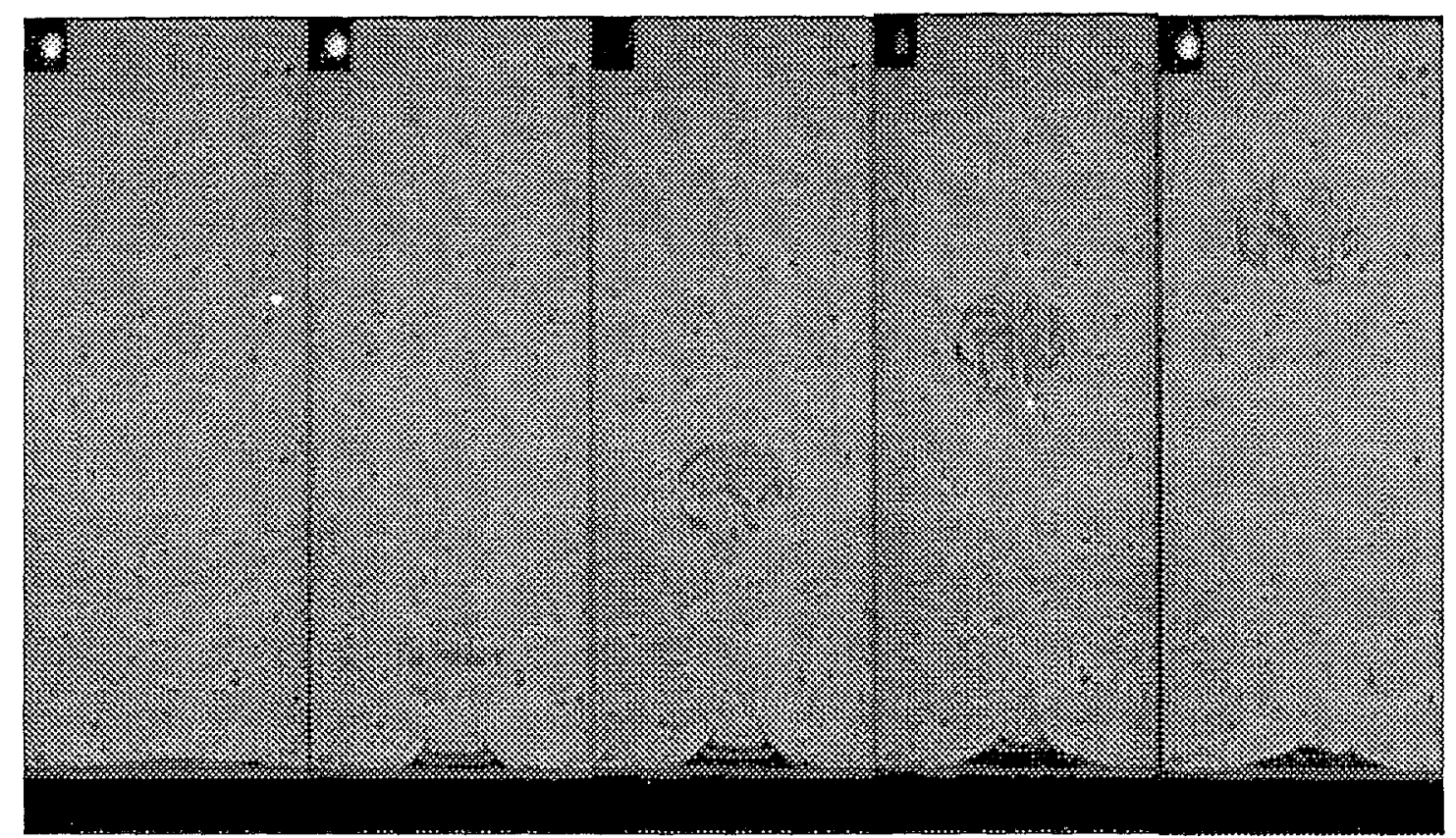

Figure 13. Top layer: Methanol, $\mathrm{H}_{2} \mathrm{O}$ solution - Bottom layer: Silicon Oil $10 \mathrm{cs}$, at $1-\mathrm{G}$ $\rho 2 / \rho 1=0.97, \mu 2 / \mu l=0.187, \mathrm{Re} \approx 1722, \mathrm{We} \approx 780, \mathrm{Oh} \approx 0.016, \Delta t \approx 0.22 \mathrm{sec}$

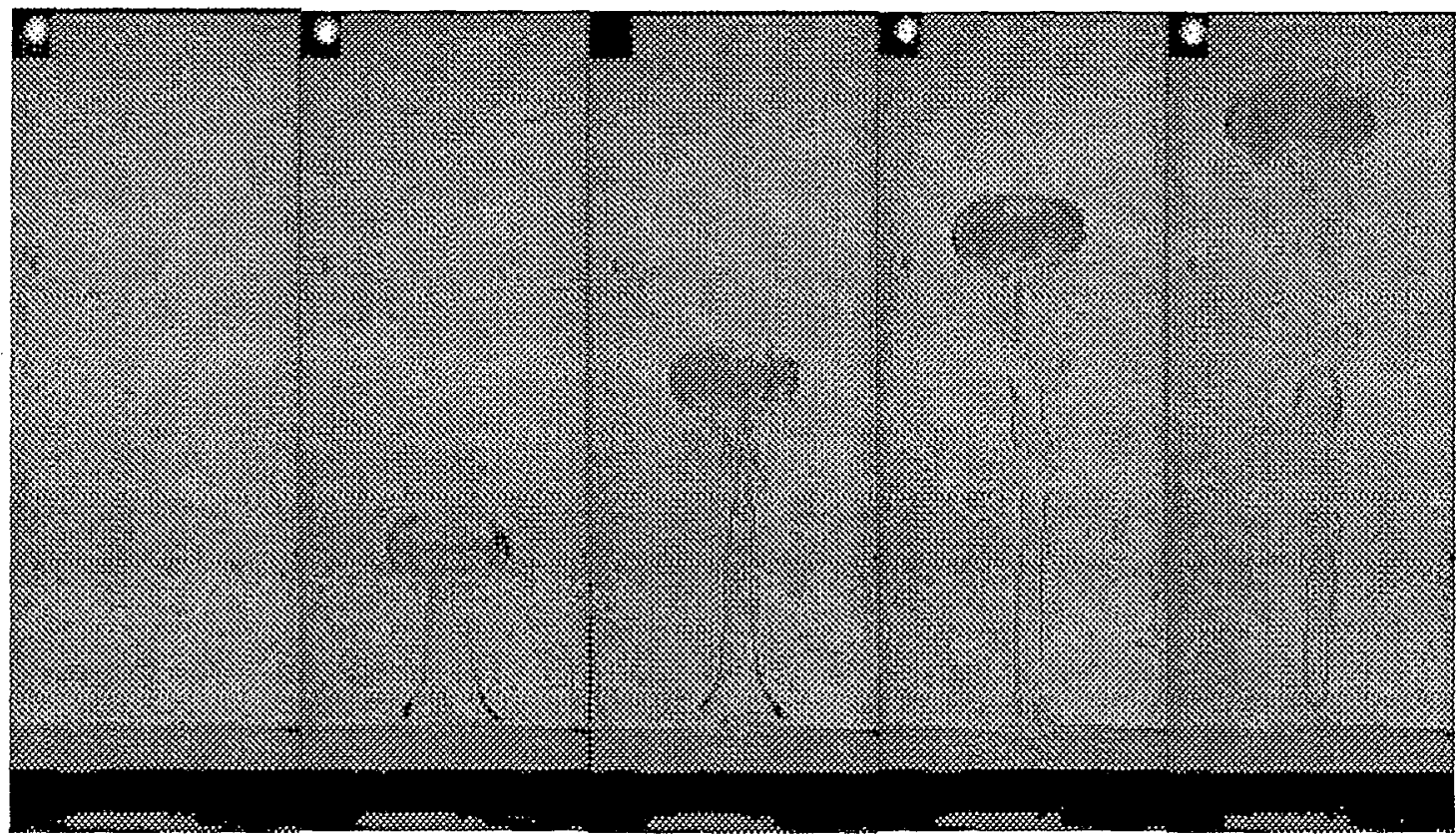

Figure 14. Top layer: Silicon Oil $10 \mathrm{cs}-$ Bottom layer: $\mathrm{H}_{2} \mathrm{O}$, at $1-\mathrm{G}$ $\rho 2 / \rho \mathrm{l}=0.94, \mu 2 / \mu \mathrm{l}=7.231, \operatorname{Re} \approx 8700, \mathrm{We} \approx 721, \mathrm{Oh} \approx 0.0019, \Delta \mathrm{t} \approx 0.22 \mathrm{sec}$ 